\title{
IN SILICO STUDIES FOR VARIOUS ANTIBACTERIAL BENZIL AND ITS SUBSTITUTED ANALOGS
}

\author{
NITHYA G ${ }^{1 *}$, SUDHA R ${ }^{1}$, BRINDHA DEVI P², CHARLES C KANAKAM ${ }^{3}$
}

${ }^{1}$ Department of Chemistry, School of Basic Sciences, Vels University, Chennai, Tamil Nadu, India. ${ }^{2}$ Department of Bioengineering, School of Engineering, Vels University, Chennai, Tamil Nadu, India. ${ }^{3}$ Department of chemistry, Formerly Presidency college, Chennai, Tamil Nadu, India. Email: nithyaranju@gmail.com

Received: 13 May 2017, Revised and Accepted: 05 September 2017

ABSTRACT

Objective: The antibacterials have moved on to low levels by more challenges toward antibacterial discovery of drug over an earlier period of 30 years. The resistance pathogens such as Staphylococcus aureus, Mycobacterium tuberculosis (MTB), and Streptococcus pneumoniae are nowadays facing difficulty in effective treatment. This leads to the necessary for the new discovery of drugs for antibacterial activity. The foremost disease in the world among all the infectious disease is found to tuberculosis (TB) which causes high proportions of mortality. Hence, we have decided on identifying the leads for the target of enzymes of infectious disease TB.

Methods: The new leads for MTB have been discovered using computer-aided drug design docking tool. The new compounds identified were made to dock into the enzyme active site retrieved from protein data bank.

Results: After three different docking strategies, the score was found to be $4.558 \mathrm{kcal} \mathrm{mol}^{-1}$ for the compound 2'-chloro-4-methoxy-3-nitro benzyl in structure activity relationship and docking studies.

Conclusion: The molecule shows valuable interactions and also it is found to be surrounded by non-polar amino acids. On further analyzing the compound it is found to be potent to antibacterial drug discovery.

Keywords: Antibacterials, Docking, Resistance, Absorption, Distribution, Metabolism, Excretion study.

(C) 2017 The Authors. Published by Innovare Academic Sciences Pvt Ltd. This is an open access article under the CC BY license (http://creativecommons. org/licenses/by/4. 0/) DOI: http://dx.doi.org/10.22159/ajpcr.2017.v10i12.19905

\section{INTRODUCTION}

The demand for novel therapeutic interventions such as antimycobacterial and antibacterial drugs is increasing daily since there are an increased drug resistant bacterial in the world market. Mycobacterium tuberculosis (MTB) which is found to be most pathogenic bacteria is a causing agent for human TB leading to a world level bacterial killer [1]. The bacterial strains for drug resistant and also multidrug-resistant existence occur using this bacteria. There are various side effects the may be caused during the treatment due to the usage of more antibiotics for curing the disease. The antibacterial activity on these pathogenic bacteria is identified by doing some preliminary studies using small dosage to reduce the side effects.

The activity was found to be superior, and it shows that benzyl and its derivatives are good antibacterial agents. As a result, the activity of pathogenic bacteria's such as Staphylococcus aureus, Klebsiella pneumonia, Escherichia coli, and Staphylococcus epidermidis gets reduced. In this study [2], the binding on specific sites for target molecules such as proteins has been carried out by a computational method, namely, virtual screening. The future point of view of the compound could also lead to the pharmacokinetic properties

\section{METHODS}

\section{Computational details}

The computational details for this study were carried out in an Intel Core I5 capacity processor with a memory of 4GB RAM running with the Windows 7 operating system. The virtual screening options for high throughput virtual screening (HTVS), standard precision (SP), and Glide extra precision (XP) docking were all checked to be executed. The module Glide XP of Schrodinger 9.3 (Glide, version 5.7, Schrodinger, LLC, New York, NY, 2015) was utilized to perform docking studies. Suitable bonding and the charges were added to the hetero atoms, and the corresponding hydrogen atoms were added to all the atoms [3].

\section{Protein preparation}

The protein file was prepared on protein preparation wizard, and the energy minimization was performed. About 500 cycles of steepest descent and 5000 cycles of conjugate gradient methods with optimized potential for liquid simulations (OPLS) 2005 force field using Schrodinger suite version 9.3 were employed. Grid-A rectangular box surrounding the active site of the protein was located using receptor grid generation panel. The "Write XP descriptor information" option was selected and "Compute RMSD" option was enabled, and rest of the parameters was kept as default. The XP Glide scoring function was used to order the bestranked compounds, and the important interactions such as $\pi$-cation and $\pi-\pi$ stacking were analyzed using XP visualizer in Glide module. The input RMSD of the crystal ligand was also ascertained [4].

\section{Preparation of ligands}

The synthesized molecules were processed through the Lipinski filters to enable the drug property. Ligand preparation was performed for the synthesized molecules using LigPrep module available in the software (LigPrep v2.2, Schrodinger LLC, New York, NY) and Epik (Epik v1.6, Schrodinger, LLC, New York, NY) to expand protonation and tautomeric states at $7.0 \pm 2.0 \mathrm{pH}$ units. Conformational sampling was also performed for all database molecules using the ConfGen search algorithm. ConfGen with OPLS 2005 force field was applied for the generation of conformers with duplicate poses eliminate if the RMSD was $<2.0 \AA$. A distance-dependent dielectric constant of 4 and maximum relative energy difference of $10 \mathrm{kcal} \mathrm{mol}^{-1}$ were applied [4].

\section{Molecular docking}

Docking studies for the synthesized compounds were performed using Glide module of Schrodinger, LLC, 2015. Primarily, using Glide 
module $[5,6]$ (Grid based ligand docking with energetics), we examined for important interactions based on the reference ligand and the protein of interest in the flexible mode docking. The Glide module with three modes of docking, HTVS, SP, and XP mode was employed sequentially. The XP mode was used for exhaustive sampling and advanced scoring, resulting in even higher enrichment.

Finally, the shortlisted hit molecules were selected based on the visual inspection of amino acid interaction, docking score and the active site cavity [7].

Absorption, distribution, metabolism, and excretion (ADME) prediction

All the synthesized compounds for our study were selected, and the molecules were subjected to ADME predicted analysis using QikProp module of Schrodinger. The important properties such as octanol water coefficient ( $\log \mathrm{P})$, human oral absorption, Lipinski's rule of five, blood brain barrier (BBB) coefficient, HERG property, and Caco-2 permeability property were predicted for the synthesized compounds, and also the predicted results were checked for any violations to determine the nature of the compounds.

\section{RESULTS AND DISCUSSION}

Synthesized compounds have taken for docking studies to establish the structure activity relationship using crystal structure of MTB cocrystallized with inhibitor thiazole benzamide (protein data bank ID:4WYC) [1]. Analysis of crystal structure of 4WYC revealed with hydrogen bonding interactions with nonpolar interaction like Trp398. The inhibitor is well associated with hydrophobic amino acids Met61, Trp398, Trp64, Tyr407, and Phe402 [8]. To validate the active site pocket the reference ligand was re-docked, and the docking score was found to be $-6.032 \mathrm{kcal} / \mathrm{mol}$. Re-docking results showed that the compound exhibited similar interactions as that of crystal structure and showed a RMSD of $1.02 \AA$ A. Further, the compounds synthesized were screened based on three different docking strategies [9]. The ligand interaction with protein was depicted in Fig. 1. The docking score and the ligand interactions for the compounds were tabulated in Table 1.

Docking studies and structure activity relationship for the compound 2'-chloro-4-methoxy-3-nitro benzil

The compound 2'-chloro-4-methoxy-3-nitro benzil was found to inhibit the pathogenic bacteria's $S$. aureus, K. pneumonia, and E. coli at a distance of $10 \mathrm{~mm}$ using disc diffusion method when compared to other compounds. The compound after three different docking strategies reveals that the score was found to be $-4.558 \mathrm{kcal} \mathrm{mol}^{-1}$. On the closer analysis of this molecule, the molecule showed similar stacking interaction like the reference molecule; the compound has also found to be surrounded by nonpolar amino acids which make this molecule potent toward antibacterial drug discovery. The binding analysis and ligand interaction diagram for the compound 2'-chloro-4-methoxy-3nitrobenzil were depicted in Fig. 2.

Docking studies and structure activity relationship for the compound 4,4'-dibromo benzil

Based on our docking studies, it has confirmed that the structure changes in the compounds series were found to be well correlated with in vitro antibacterial results. The compound 4,4'-dibromo benzil possessed high docking score of $-5.225 \mathrm{kcal} / \mathrm{mol}$ with the stacking interaction with amino acid Phe402. The activity of this compound also found to be well correlated with the reference ligand. The close analysis of this compound revealed that the compound is well packed with nonpolar interactions which make this compound more active against the pathogenic bacteria. The binding analysis and ligand interaction diagram for the most active compound 4, 4'-dibromo benzil are shown in Fig. 3. The docking score and its ligand interaction with the synthesized compounds are tabulated in Table 1.

Docking studies and structure activity relationship for the compound 2,2'-dichloro benzil

The compound 2,2'-dichloro benzil was found to inhibit the bacteria at a distance of $8 \mathrm{~mm}$. This is quite lesser than the other molecules. This makes this molecule more effective binding, and the docking score was found to be $-4.523 \mathrm{kcal} \mathrm{mol}^{-1}$. The binding analysis of this compound reveals that the compound well fitted into the active site pocket and

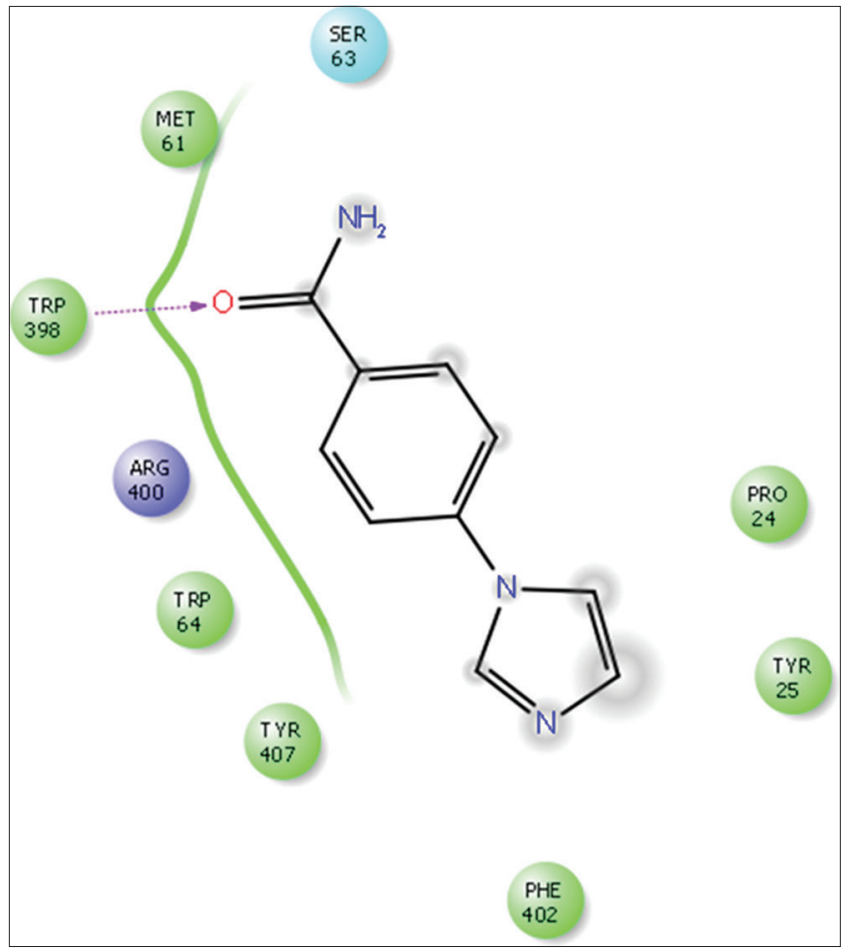

Fig. 1: Reference ligand interaction with protein

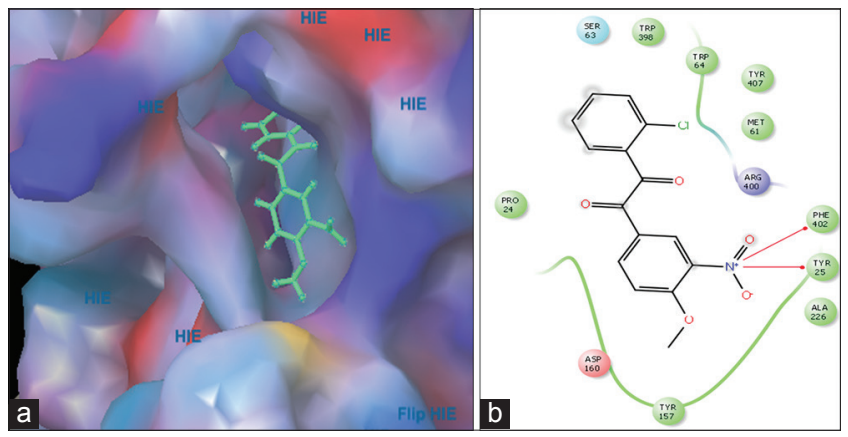

Fig. 2: ( $a$ and b) Binding analysis and ligand interaction diagram for the most active compound 2'-chloro-4-methoxy-3-nitro benzil

Table 1: Docking score and ligand interaction results for the synthesized compounds

\begin{tabular}{|c|c|c|c|}
\hline S. No. & Compound name & Docking score kcal/mol & Ligand interaction \\
\hline 1. & 2'-chloro-4-methoxy-3- nitro benzil & -4.558 & Phe402, Tyr25 \\
\hline 2. & 4'4-dibromo benzil & -5.225 & Phe402 \\
\hline 3. & 2,2'-dichlorobenzil & -4.523 & Trp64, Arg400 \\
\hline 4. & Benzil & -3.140 & Trp64, Arg400 \\
\hline
\end{tabular}


Table 2: ADME prediction for the synthesized compounds

\begin{tabular}{|c|c|c|c|c|c|}
\hline \multirow[t]{2}{*}{ Compound name } & \multirow{2}{*}{$\frac{\text { QPlog }}{\text { Po/w }}$} & \multirow{2}{*}{$\frac{\text { QPlog }}{\text { HERG }^{b}}$} & \multirow{2}{*}{$\frac{\mathrm{QPP}}{\text { Caco }^{\mathrm{c}}}$} & \multirow{2}{*}{$\frac{\text { QPlog }}{\text { BB }^{\mathrm{d}}}$} & \multirow[t]{2}{*}{ Percent human oral absorption } \\
\hline & & & & & \\
\hline 2'-chloro-4-methoxy-3-nitro benzil & 3.112 & -5.016 & 1545.995 & -0.513 & 100 \\
\hline 2,2'-dichlorobenzil & 4.143 & -2.686 & 314.014 & -0.359 & 98.291 \\
\hline 4,4'-dibromo benzil & 4.153 & -3.285 & 202.689 & -0.363 & 96.543 \\
\hline Benzil & 2.98 & -3.193 & 202.314 & -0.652 & 82.365 \\
\hline
\end{tabular}

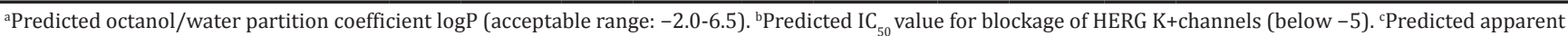
Caco-2 cell permeability in $\mathrm{nm} / \mathrm{s}(<25$ poor; $>500$ great $)$. dPredicted brain/blood partition coefficient $(-3.0-1.2)$. ${ }^{\text {ePercent }}$ human oral absorption $(<25 \%$ is poor and $>80 \%$ is high). ${ }^{\mathrm{f}} \mathrm{Rule}$ of 5 violation (mol_MW $<500, \mathrm{QP} \operatorname{logPo} / \mathrm{w}<5$, donorHB $\leq 5$, accptHB $\leq 10$ ). ADME: Absorption, distribution, metabolism, and excretion

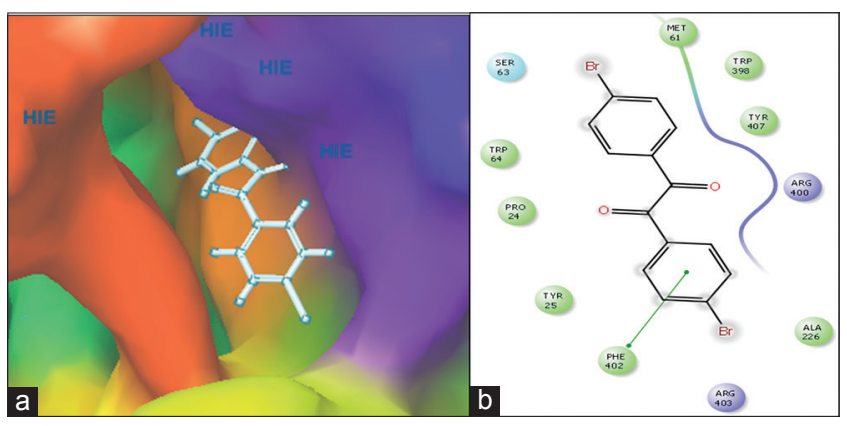

Fig. 3: (and b) Binding analysis and ligand interaction diagram for the most active compound 4,4'-dibromo benzil

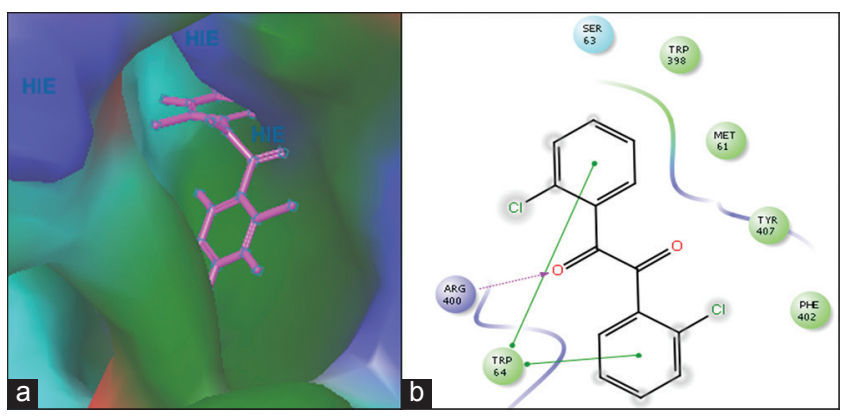

Fig. 4: (a and b) Binding analysis and ligand interaction diagram for the most active compound 2,2'-dichloro benzyl

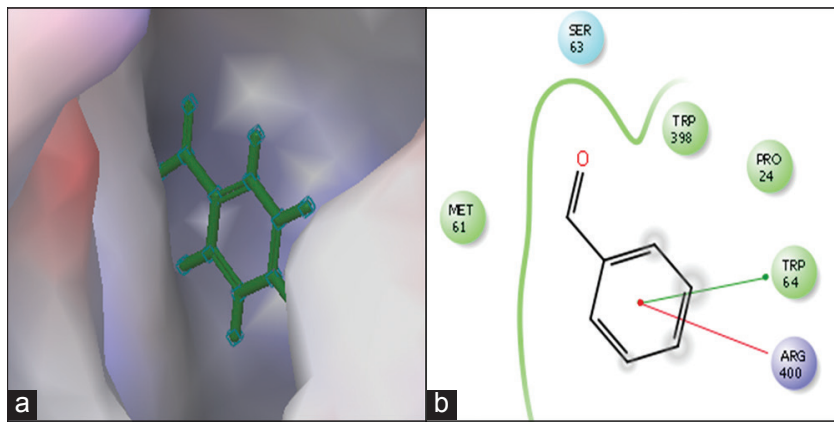

Fig. 5: ( $a$ and b) Binding analysis and ligand interaction diagram for the most active compound benzil

the group phenyl chloride was found to interact with nonpolar amino acids Trp64 and Arg400 which reveals that there are two stacking interactions making this compound more stable for further processing as better drug compound [10]. The binding analysis and ligand interaction for the compound 2,2'-dichloro benzil was depicted in Fig. 4.

Docking studies and structure activity relationship for the compound benzil

The compound benzil was found to inhibit the bacteria $K$. pneumoniae at a distance of $12 \mathrm{~mm}$. This compound after in silico screening analysis was found to possess good docking score $-3.140 \mathrm{kcal} \mathrm{mol}^{-1}$. On the closer analysis of this compound reveals that the molecule has well fitted into the active site pocket of the protein; also their ligand interaction shows that the molecule was surrounded by nonpolar amino acid and it is found to be interact with an amino acid Trp64 and Arg400 which is an important interaction of original ligand [11,12]. The binding analysis and ligand interaction diagram for the compound benzil were depicted in Fig. 5 .

\section{ADME prediction}

To further account for the potential of the compounds to act as efficient drug candidates, their ADME properties were also calculated in silico using Qikprop. The obtained values for molecular logP, HERG property, Caco accessibility, BBB, and human oral absorption; it is also used to assess violation of Lipinski's rule of five if any. All the compounds were shown to correlate well with the human oral absorption. BBB separates the human brain from the direct contact of the circulatory system, thus protecting the brain for unwanted solute particles. Both the predicted compounds were shown to be BBB negative ensuring their administration safe for the brain. The ADME predictions for the synthesized compounds were tabulated in Table 2

\section{CONCLUSION}

The structured based drug design strategy had been carried out by medicinal chemistry tools. The new scaffold molecules have been identified using docking studies. This strategy revealed hitherto unknown binding pockets and inhibitor binding modes distinct from the earlier reported inhibitors and will be exploited successfully in further antimycobacterial drug development process. The compound 2'-chloro4-methoxy-3-nitro benzil was found to be most active in both in silico and in vitro antibacterial analysis. Further, these compounds will be carried out for their antimycobacterial property as these are small molecule leads could easily cross the cell barrier systems in mycobacteria.

\section{ACKNOWLEDGMENT}

Authors thank the Department of Chemistry and Biotechnology Engineering, Vels University, for providing us the computer system with all the configuration which we needed for our study.

\section{REFERENCES}

1. Koul A, Arnoult E, Lounis N, Guillemont J, Andries K. The challenge of new drug discovery for tuberculosis. Nature 2011;469(7331):483-90.

2. Hajduk PJ, Greer J. A decade of fragment-based drug design: Strategic advances and lessons learned. Nat Rev Drug Discov 2007;6:211-9.

3. Cuomow AM. Maestro, Schrödinger. Version 9.3. New York, NY: Limited Liability Company (LLC); 2015.

4. Saxena S, Devi PB, Soni V, Yogeeswari P, Sriram D. Identification of novel inhibitors against Mycobacterium tuberculosis L-alanine dehydrogenase (MTB-AlaDH) through structure-based virtual screening. J Mol Graph Model 2014;47:37-43.

5. Friesner RA, Banks JL, Murphy RB, Halgren TA, Klicic JJ, Mainz DT. Gilde: A new approach for rapid, accurate docking accuracy: Method and assessment of docking accuracy. J Med Chem 2004;47(7):1739-49.

6. Kawatkar S, Wang H, Czrminski R, Joseph-Mclarthy D. Virtual fragment screening: An exploration of various docking and scoring protocols for fragments using glide. J Comput Aided Mol Des 2009;23:527-39.

7. Alverez J, Shoichet B, editors. Virtual Screening in Drug Discovery. 
Boca Raton, Florida: Taylor Francis; 2005

8. Dai R, Geders TW, Liu F, Park SW, Schnappinger D, Aldrich CC, et al. Fragment-based exploration of binding site flexibility in Mycobacterium tuberculosis bioa. J Med Chem 2015;58:5208-17.

9. Sudha R, Kanakam CC, Nithya G. Synthesis, characterization and antimicrobial activity of substituted benzilic acids. Chem Tech 2015;8(10):383-7.

10. Jennings A, Tennant M. Discovery strategies in a bio pharmaceutical start up: Maximising your chances of success using computational filters. Curr Pharm Des 2005;11:335-44.

11. Duttaa S, Rayb S, Nagarajanc K. Docking study of some glutamic acid derivatives as potent antineoplastic agents. Int J Pharm Pharm Sci 2014;6(4):419-22

12. Sharma RB, Chetia D. Docking studies on quinine analogy for plasmepsin-II of malaria parasite using bioinformatics tools. Int $\mathrm{J}$ Pharm Pharm Sci 2013;5(3):681-5. 